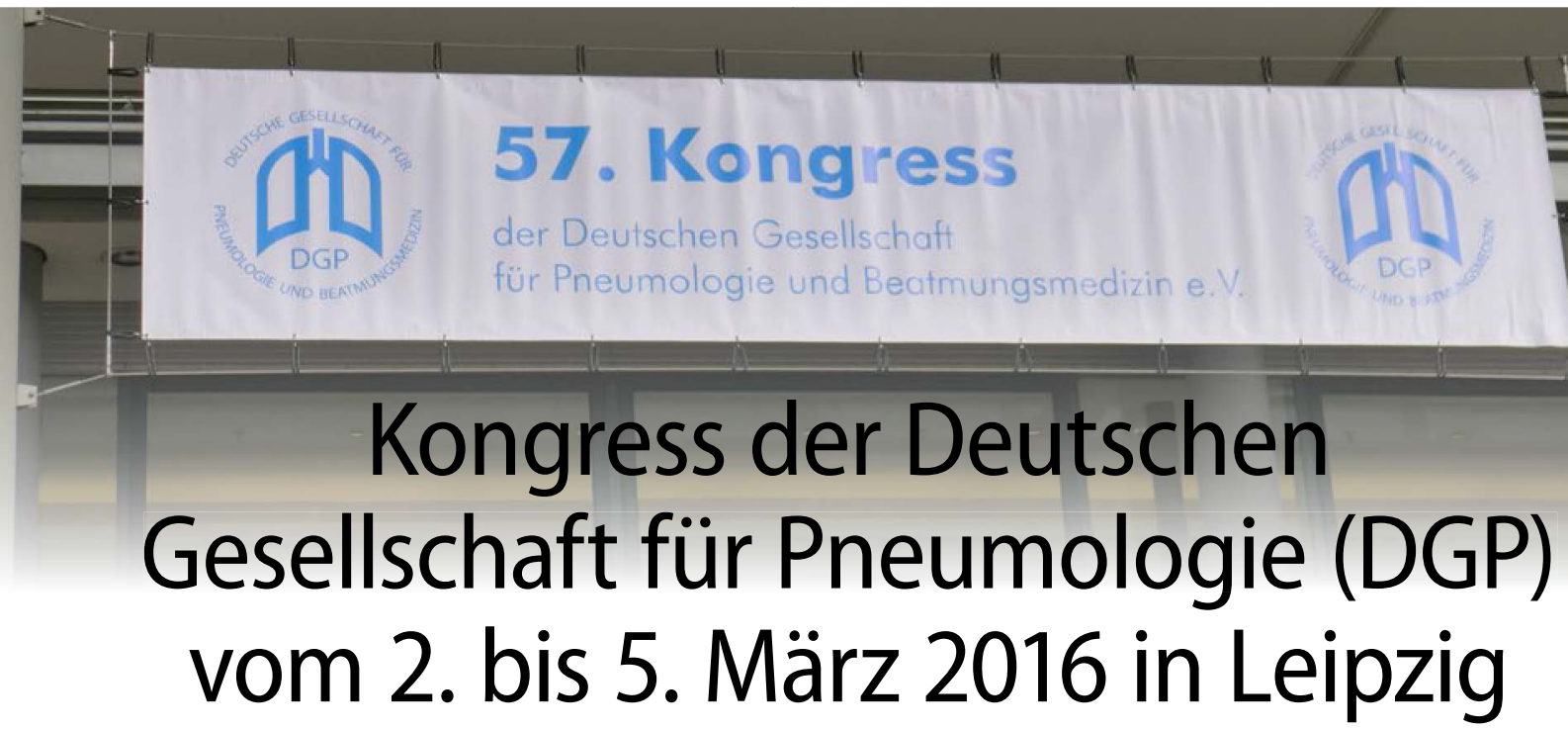

Innovative Pneumologie

\title{
Große Fortschritte in der Therapie von Lungenerkrankungen
}

\author{
Unter dem Motto "Innovative Pneumologie" tagen Lungenfachärzte in den ersten Märztagen in \\ Leipzig. Highlights des DGP-Jahreskongresses waren neuartige Therapiekonzepte bei Lungen- \\ krebs, -fibrose oder -hochdruck sowie die pneumologischen Aspekte in der Flüchtlingsmedizin.
}

An die 4.000 Teilnehmer, über 230 Veranstaltungen sowie eine ausgebuchte Industrieausstellung verdeutlichen eindrucksvoll, dass sich die Pneumologie in den letzten 15 bis 20 Jahren zu einem großen internistischen Fach entwickelt habe, unterstrich Tagungspräsident Prof. Hubert Wirtz, Direktor der Medizinischen Klinik und Poliklinik I am Universitätsklinikum Leipzig.

Damit wird dem Umstand Rechnung getragen, dass Atemwegserkrankungen eine zunehmende Bedeutung erlangen in den Morbiditäts- und Mortalitätsstatistiken. Auf vielen Gebieten - Asthma, COPD, Lungenentzündung, Schlafapnoe-Syndrom oder Lungenkrebs - gibt es wesentliche Therapiefortschritte.

\section{Fortschritt beim Lungenkrebs}

nach Jahrzehnten des Stillstandes

Beispiel Lungenkrebs: Da die Erkrankung häufig erst im metastasierten Stadium diagnostiziert wird, konnte die Medizin den meisten Patienten über Jahrzehnte keine Therapieverbesserungen bieten. Über 40.000 Menschen sterben jedes Jahr in Deutschland an diesem besonders tödlichen Malignom.

„Doch der Fortschritt hat begonnen“, so Wirtz. Zunehmend werden in bestimmten Krebsuntereinheiten molekulare Alterationen von Signalwegen entdeckt, die gezielt behandelt werden können.

Hinzu kommt, dass endlich das körpereigene Immunsystem zur Tumorabwehr genutzt werden kann. Denn, so Wirtz, man habe Mechanismen entdeckt, mit denen sich der Tumor dem Zugriff des Immunsystems entzieht. In diesen Prozess greifen die Checkpoint-Inhibitoren ein. Bei einem Teil der zuvor nicht behandelbaren Patienten gelingt es mit diesen Medikamenten, eine länger anhaltende Remission zu erzielen. Es ist ein Silberstreif am Horizont, kommentierte Wirtz.

\section{Therapieansätze gegen}

\section{schleichende Belastungsdyspnoe}

Mehrere neue Therapieoptionen gibt es auch für zwei Lungenerkrankungen mit anfangs sehr diskreter Symptomatik, die häufig erst auffällt, wenn die Patienten unter Alltagsbelastungen in Atemnot geraten: Die Lungenfibrose und die pulmonale arterielle Hypertonie (PAH). Beide Erkrankungen führen unbehandelt binnen weniger Jahre zum Tod.

Gegen die PAH haben wir heute vier bis sechs medikamentöse Strategien, die wir kombinieren können, erläutert Wirtz. Dadurch gelingt es, die Symptome zu reduzieren und die Prognose zu verbessern.

Zur Therapie der Lungenfibrose wurden zuletzt zwei Medikamente zugelassen. Eine Heilung ist nicht möglich, aber es gelingt, das Fortschreiten der Erkrankung um etwa 50\% abzubremsen. Das kann für Patienten einen erheblichen Unterschied zum natürlichen Verlauf bedeuten.

Ein weiterer Fortschritt betrifft die Diagnostik: Mithilfe sogenannter elektronischer Nasen können heute pathologische Moleküle in der Atemluft detektiert werden. Die Nanosensoren würden nicht einzelne Moleküle erkennen, aber bestimmte Muster, erklärt Wirtz, die eine Differenzierung zwischen gesund und krank mit erstaunlicher Sicherheit ermöglichen. Speziell ausgebildete Hunde können das mit ihrem feinen Geruchssinn. Wirtz ist überzeugt, dass die Nanosensoren-Technologie in $\mathrm{Zu}$ kunft weiter an Bedeutung gewinnt. 